\title{
Replication of GWAS Coding SNPs Implicates MMEL1 as a Potential Susceptibility Locus among Saudi Arabian Celiac Disease Patients
}

\author{
Omar I. Saadah, ${ }^{1,2}$ Noor Ahmad Shaik, ${ }^{3}$ Babajan Banaganapalli, ${ }^{2}$ Mohammed A. Salama, ${ }^{2,4}$ \\ Sameer E. Al-Harthi, ${ }^{2,5}$ Jun Wang, ${ }^{2,6}$ Harbi A. Shawoosh, ${ }^{7}$ Sharifa A. Alghamdi, \\ Yagoub Y. Bin-Taleb, ${ }^{1}$ Bakr H. Alhussaini, ${ }^{1}$ Ramu Elango, ${ }^{2}$ and Jumana Y. Al-Aama ${ }^{2,3}$ \\ ${ }^{1}$ Department of Pediatrics, Faculty of Medicine, King Abdulaziz University, Jeddah 21589, Saudi Arabia \\ ${ }^{2}$ Princess Al-Jawhara Al-Brahim Center of Excellence in Research of Hereditary Disorders, King Abdulaziz University, \\ Jeddah, Saudi Arabia \\ ${ }^{3}$ Department of Genetic Medicine, Faculty of Medicine, King Abdulaziz University, Jeddah, Saudi Arabia \\ ${ }^{4}$ Department of Biology, Faculty of Science, King Abdulaziz University, Jeddah, Saudi Arabia \\ ${ }^{5}$ Department of Pharmacology, Faculty of Medicine, King Abdulaziz University, Jeddah, Saudi Arabia \\ ${ }^{6}$ BGI, Beishan Industrial Zone, Yantian District, Shenzhen 518083, China \\ ${ }^{7}$ Department of Pediatrics, King Fahd Armed Forces Hospital, Jeddah, Saudi Arabia \\ ${ }^{8}$ Department of Pediatrics, Division of Gastroenterology, King Faisal Specialist Hospital and Research Center, Jeddah, Saudi Arabia
}

Correspondence should be addressed to Jumana Y. Al-Aama; jalama@kau.edu.sa

Received 25 August 2015; Accepted 16 November 2015

Academic Editor: George Perry

Copyright (c) 2015 Omar I. Saadah et al. This is an open access article distributed under the Creative Commons Attribution License, which permits unrestricted use, distribution, and reproduction in any medium, provided the original work is properly cited.

Celiac disease (CD), a gluten intolerance disorder, was implicated to have 57 genetic susceptibility loci for Europeans but not for culturally and geographically distinct ethnic populations like Saudi Arabian CD patients. Therefore, we genotyped Saudi CD patients and healthy controls for three polymorphisms, that is, Phe196Ser in IRAK1, Trp262Arg in SH2B3, and Met518Thr in MMEL1 genes. Single locus analysis identified that carriers of the $518 \mathrm{Thr} / \mathrm{Thr}$ (MMEL1) genotype conferred a 1.6-fold increased disease risk compared to the noncarriers $(\mathrm{OR}=2.6 ; 95 \% \mathrm{CI}: 1.22-5.54 ; P<0.01)$. This significance persisted even under allelic $(\mathrm{OR}=1.55$; 95\% CI: $1.05-2.28 ; P=0.02)$ and additive ( $\mathrm{OR}=0.35 ; 95 \% \mathrm{CI}: 0.17-0.71 ; P=0.03)$ genetic models. However, frequencies for Trp262Arg (SH2B3) and Phe196Ser (IRAK1) polymorphisms were not significantly different between patients and controls. The overall best MDR model included Met518Thr and Trp262Arg polymorphisms, with a maximal testing accuracy of $64.1 \%$ and a maximal cross-validation consistency of 10 out of $10(P=0.0156)$. Allelic distribution of the $518 \mathrm{Thr} / \mathrm{Thr}$ polymorphism in MMEL1 primarily suggests its independent and synergistic contribution towards CD susceptibility among Saudi patients. Lack of significant association of IRAK and SH2B3 gene polymorphisms in Saudi patients but their association in European groups suggests the genetic heterogeneity of CD.

\section{Introduction}

Celiac disease $(\mathrm{CD})$ is a T-cell-mediated inflammatory disorder with underlying involvement of autoimmune, genetic, and environmental components [1,2]. This chronic enteropathy is caused by permanent intolerance to ingested gluten from wheat, barley, and rye in genetically susceptible children and adults [3-5]. The common clinical manifestations presented by patients with $\mathrm{CD}$ are chronic diarrhea, abdominal distension, failure to thrive, and short stature $[4,6]$. An approximate $1 \%$ prevalence rate of $\mathrm{CD}$ among Caucasians places them as the most vulnerable ethnic group for developing CD. However, current day improved knowledge about celiac disease prevalence and clinical and diagnostic characteristics has indicated that it is common not only in Europe, but also in developing countries where wheat is 
included as a major dietary component [4, 7]. In the Middle East, recent trends indicate the variable prevalence of $\mathrm{CD}$ from $0.14 \%$ to $1.17 \%$ in low risk and from $2.4 \%$ to $44 \%$ in high risk populations of Arab origin [8-10].

Celiac disease is a complex pathology where disease onset is governed by various genetic factors. The genetic liability to $\mathrm{CD}$ is evidenced by the higher rate of familial incidence and also by its strict linkage with some human leukocyte antigens (HLA) class 2 alleles [11]. Polymorphic HLA-DQ2 and/or HLA-DQ8 alleles have been identified as a prerequisite but they alone cannot justify the development of CD. Therefore, it is expected that non-HLA genetic loci play a role in celiac disease susceptibility. The introduction of genome-wide association studies (GWAS) and subsequent immunochip fine mapping studies has been a great breakthrough in bringing down the celiac disease risk loci (both HLA and non-HLA) to 57 SNPs. Together all these risk loci can only explain up to $54 \%$ of celiac disease risk leaving a subset of the genetic risk factors for celiac disease unexplained among certain case groups [12-18].

A common complication faced by geneticists in deriving GWAS based genotype-phenotype associations for complex traits like CD is that most of these studies were conducted in European patients, so they miss population-specific genetic effects in culturally and ethnically different disease groups. In addition to this GWAS sample sets consist of nonuniform control cohorts with differential contribution to disease association signal. The prediction of clinical relevance for disease associated GWAS loci is further complicated by the fact that the majority of SNPs are located in intronic or regulatory regions and they have no readily annotated gene function and therefore cannot be assigned to a particular biological pathway. On the contrary, SNPs occurring in coding regions are often able to alter amino acid sequence of polypeptides which in turn results in structural and functional changes in the protein encoded, hence the cellular functions. An important finding revealed by Trynka et al. [16] is that, on functional annotation of 57 genetic loci associated with celiac disease, only 3 genetic variations, that is, rs1059702 in IRAK1, rs3184504 in SH2B3, and rs3748816 in MMEL1, are found to occur in exonic regions and subsequently affect the amino acid sequences of concerned proteins.

The clinical ascertainment of real disease causal genetic variations from statistically correlated CD-GWAS loci requires data from population-specific replication studies. It is well agreed that conducting genetic studies in the mostly consanguineous Arabian population has a great potential to identify novel genetic connections and may also narrow down the number of real disease associated genes in complex diseases. To the best of our information, until today no study has examined the contribution of genetic variations in celiac disease among patients of Saudi Arabian origin. Therefore, the current study has aimed to replicate the association of three amino acid substitution SNPs identified by fine mapped loci of GWAS results of European populations to question their implication in CD development among Saudi Arabian patients.

\section{Methods}

2.1. Subjects and Sampling. Ethics approval for the present study was obtained from the Research Ethics Committee, King Abdulaziz University Hospital, Jeddah. Study participants comprised Saudi nationals of whom 97 were unrelated patients with celiac disease and 124 were self-reported healthy controls, who never reported any health complications including allergies. Diagnosis of celiac disease was made by testing for antibodies against gliadin and endomysium (EMA) or tissue transglutaminase (TGA) as well as a confirmatory small bowel biopsy as per the criteria set out by the European Society for Paediatric Gastroenterology, Hepatology and Nutrition (ESPHGAN). $2 \mathrm{~mL}$ of blood samples was collected for genetic analysis from each participant after obtaining their consent or approval.

2.2. Genetic Analysis. The DNA was isolated from whole blood samples by using a DNA blood mini kit (QIAamp, Qiagen, Inc., Valencia, CA, USA) following the manufacturer's instructions. Three SNPs, that is, rs1059702, rs3184504, and rs3748816, were genotyped using commercial TaqMan allelic discrimination assays (Applied Biosystems, Foster City, California, USA) on an ABI7500HT sequence detection system (Applied Biosystems). The TaqMan probe labeling is as follows: VIC dye (green fluorophore) was made correspondent to wild type allele, whereas FAM dye (blue fluorophore) was made for mutant allele. The emission of green color fluorescence during amplification cycle indicates homoallelic native type, blue color indicates homoallelic mutant type, and combination of both blue and green color fluorescence indicates the heteroallelic state. The scatter plot was prepared based on the automatically computed threshold cycle values of each sample corresponding to the signal of either FAM or VIC. The $10 \mu \mathrm{L}$ reaction mixture consisting of $5 \mu \mathrm{L}$ of TaqMan Genotyping Master Mix (which contains AmpliTaq Gold DNA Polymerase UP (Ultra Pure), dNTPs without dUTP, Passive Reference ROX dye, and optimized mix components), $0.25 \mu \mathrm{L}$ TaqMan SNP Genotyping Assay (which includes $300 \mathrm{nM}$ of each specific oligonucleotide primer and MGB probe $200 \mathrm{nM})$, and $1 \mu \mathrm{L}$ of DNA $(50 \mathrm{ng} / \mu \mathrm{L})$ was carried out in a Micro Amp optical 96-well reaction plate. The conditions for PCR included $60^{\circ} \mathrm{C}$ for $1 \mathrm{~min}$ and $95^{\circ} \mathrm{C}$ for $10 \mathrm{~min}$ followed by 40 cycles of $95^{\circ} \mathrm{C}$ for $15 \mathrm{~s}$ and $60^{\circ} \mathrm{C}$ for $1 \mathrm{~min}$. Genotyping calling was done using SDS version 2.3 software.

2.3. Statistical Analysis. Statistical analyses were performed with GraphPad QuickCalcs, version 6.0 (GraphPad Software Inc., USA), online statistical software. For each SNP, allele and genotype frequencies were computed in celiac patients and controls. Statistically significant difference in allele and genotypes was determined using Pearson's standard chi-squared test, odds ratio (OR), and 95\% confidence interval (CI). Hardy-Weinberg equilibrium was tested on a contingency table of observed versus predicted genotype distributions using a chi-squared test with one degree of freedom. The epistasis between three investigated polymorphisms and incidence of CD was examined by nonparametric and genetic model-free multifactor dimensionality reduction 
TABLE 1: Characteristics of nonsynonymous SNPs screened in celiac patients.

\begin{tabular}{lcccccccc}
\hline Gene symbol & Gene name & RS ID & Alleles & $\begin{array}{c}\text { Minor allele } \\
\text { frequency }\end{array}$ & $\begin{array}{c}\text { cDNA } \\
\text { location }\end{array}$ & $\begin{array}{c}\text { Genomic } \\
\text { location }\end{array}$ & Exon & Protein effect \\
\hline IRAK1 & $\begin{array}{c}\text { Interleukin-1 } \\
\text { receptor-associated kinase 1 }\end{array}$ & rs1059702 & A/G & 0.37 & c.587 A > G & X:154018741 & 5 & Phe196Ser \\
\hline SH2B3 & SH2B adaptor protein 3 & rs3184504 & T/C & 0.22 & c.784 T > C & $12: 111446804$ & 2 & Trp262Arg \\
\hline MMEL1 & $\begin{array}{c}\text { Membrane } \\
\text { metalloendopeptidase-like 1 }\end{array}$ & rs3748816 & T/C & 0.46 & c.1553 T > C & $1: 2595307$ & 16 & Met518Thr \\
\hline
\end{tabular}

(MDR) data mining approach using open-source multifactor dimensionality reduction (MDR) software (MDR version 2.0 beta 5, http://www.epistasis.org/). Statistical significance was tested through 1,000 permutation tests (MDR permutation testing module 0.4.9 alpha) to compare observed testing accuracies with those expected under the null hypothesis of null association. A $P$ value of 0.05 was considered statistically significant.

\section{Results}

Our study group comprised 57 adolescent and 40 childhood celiac disease patients. Gender distribution of disease group showed that females $(54 \%)$ were slightly higher in number compared to their male counterparts. The mean age of disease onset among adults was $16 \pm 4.9$ years and in children it was $9.6 \pm 4.9$ years. The control group consisted of 140 adults, whose mean age was $29 \pm 3$ years. The common clinical complaints seen in celiac patients were as follows: chronic diarrhea (82\%), anorexia (80\%), abdominal distension (75\%), and abdominal pain (31\%). Among the autoimmune disorders that were presented in our CD group were type 1 diabetes mellitus (32\%), autoimmune thyroiditis $(8 \%)$, and systemic lupus erythematosus (3\%). The other nonautoimmune mediated disorders seen in patient group were osteomalacia (5\%), seizure disorders (4\%), and Down syndrome (4\%).

Regarding genotyping results, all the three tested SNPs (rs1059702, rs3184504, and rs3748816) were compatible to Hardy-Weinberg equilibrium in both CD patients and controls. The frequencies of the AA (Phe/Phe), AG (Phe/Ser), and GG (Ser/Ser) genotypes for IRAK1 rs1059702 SNP among celiac cases in this study were $23.71 \%, 24.74 \%$, and $51.54 \%$, respectively (Table 1).

The minor allele frequency of A was found to be 36.08 . However, the celiac patients and healthy controls did not significantly differ in terms of their genotype distribution $(P=0.72)$ and allele distributions $(P=0.28)$ (Table 2). For SH2B3 SNP, frequencies of TT (Trp/Trp), TC (Trp/Arg), and CC (Arg/Arg) genotypes and T (Trp) and C (Arg) alleles were found to be insignificantly different between both the study groups when tested for recessive and dominant models of disease risk $(P \geq 0.51)$. The genotypes encoding Met/Met (TT), Thr/Met (TC), and Thr/Thr (CC) in MMEL1 rs3748816 SNP have indicated that $\mathrm{Thr} / \mathrm{Thr}$ genotype was significantly different between celiac cases (25.77\%) and controls (12.09\%) $[P=$ $0.01 ; \mathrm{OR}(95 \% \mathrm{CI})=2.60(1.22-5.54)]$. In correspondence to this finding, the dominant model analysis has also revealed a significant association of $\mathrm{Thr} / \mathrm{Thr}$ genotypes over Met/Met $+\mathrm{Thr} /$ Met genotypes with celiac disease risk $[P=0.003$, OR $(95 \% \mathrm{CI})=0.35(0.17-0.71)]$. The minor allele $\mathrm{C}$ coding for Thr was significantly higher among celiac cases compared to controls $[P=0.02$, OR $(95 \% \mathrm{CI})=1.55(1.05-2.28)]$.

To further elaborate our genotype findings, MDR analysis was applied to characterize high order gene-gene interactions in cases and controls. Table 3 summarizes the results of exhaustive MDR analysis for combinations of the three polymorphisms studied for the risk of developing celiac disease. The best models will be accompanied by their testing accuracy, cross-validation consistency, and significant level determined by permutation testing. MDR results have revealed the interactions between MMEL1 (c.1553 T > C; p.Met518Thr) and SH2B3 (c.784 T > C; p.Trp262Arg) polymorphisms as the overall best model included with maximal testing accuracy of $63.18 \%$ and a maximal cross-validation consistency of 10 out of 10 . This model was significant at the 0.0156 level. These results have reinforced the significant results of our single locus analysis, that is, MMEL1 as a risk factor for CD development in the Saudi Arabian population.

\section{Discussion}

The recent GWAS have implicated numerous genetic loci in celiac disease predisposition [12, 13, 17, 19]. However, genetic variations (in terms of allele frequency and linkage disequilibrium) and environmental factors (in terms of dietary patterns, alcohol, and smoking) often vary across different ethnicities and regions resulting in lack of consensus regarding the association of celiac disease susceptibility SNPs. Therefore, in the present work, we investigated the association of three nonsynonymous polymorphisms in MMEL1, IRAK1, and SH2B3 genes to enhance the likelihood of identifying alleles that are assumed to have functionally deleterious consequences with subsequent celiac disease risk predisposition in the Saudi Arabian population. The principle finding was that rs3748816 in MMEL1 is a significant contributor to celiac disease susceptibility in patients of Saudi Arabian ancestry. Although allele and genotype frequencies of IRAK1 and SH2B3 were insignificantly different between patients and controls, a potential synergistic effect was seen between MMEL1 and SH2B3 genes, reinforcing the significance of MMEL1 in celiac disease susceptibility. The compliance of three examined genetic polymorphisms with HardyWeinberg equilibrium in both patients and controls suggests that our results are unlikely to be biased by genotyping errors or population stratification. 
TABLE 2: Genotype distributions and allele frequencies of examined polymorphism between patients and controls, as well as their prediction for celiac disease risk.

\begin{tabular}{|c|c|c|c|c|c|c|c|}
\hline Gene & SNP ID & Genotype/allele & Cases (\%) & Controls (\%) & $x^{2}$ & $P$ & OR (95\% CI) \\
\hline \multirow{7}{*}{ IRAK1 } & \multirow{7}{*}{ rs1059702 } & AA & $23(23.71)$ & $32(25.8)$ & Reference & & \\
\hline & & AG & $24(24.74)$ & $38(30.64)$ & 0.1162 & 0.7321 & $0.87(0.419-1.84)$ \\
\hline & & GG & $50(51.54)$ & $54(43.54)$ & 0.5639 & 0.4539 & $1.28(0.66-2.49)$ \\
\hline & & GG versus $A G+A A$ & \# & \# & 0.12 & 0.72 & $1.11(0.60-2.07)$ \\
\hline & & AA versus AG + GG & $\#$ & $\#$ & 1.3 & 0.23 & $0.72(0.42-1.23)$ \\
\hline & & $\mathrm{A}$ & $70(36.08)$ & $102(41.12)$ & Reference & & \\
\hline & & G & $124(63.91)$ & $146(58.87)$ & 1.164 & 0.2815 & $1.238(0.840-1.82)$ \\
\hline \multirow{7}{*}{$\mathrm{SH} 2 \mathrm{~B} 3$} & \multirow{7}{*}{ rs3184504 } & TT & $58(59.79)$ & $75(60.48)$ & Reference & & \\
\hline & & TC & $36(37.11)$ & $42(34.67)$ & 0.077 & 0.78164 & $1.08(0.618-1.895)$ \\
\hline & & $\mathrm{CC}$ & $3(3.09)$ & $5(4.83)$ & 0.361 & 0.54813 & $0.64(0.155-2.695)$ \\
\hline & & $\mathrm{TT}$ versus $\mathrm{TC}+\mathrm{CC}$ & \# & \# & 0.01 & 1 & $1.02(0.59-1.77)$ \\
\hline & & $\mathrm{CC}$ versus $\mathrm{TC}+\mathrm{TT}$ & $\#$ & $\#$ & 0.42 & 0.51 & $1.59(0.38-6.54)$ \\
\hline & & $\mathrm{T}$ & $152(78.3)$ & $192(78.68)$ & Reference & & \\
\hline & & $\mathrm{C}$ & $42(21.7)$ & $52(21.31)$ & 0.018 & 1 & $0.97(0.616-1.527)$ \\
\hline \multirow{7}{*}{ MMEL1 } & \multirow{7}{*}{ rs3748816 } & TT & $38(41.23)$ & $55(44.35)$ & Reference & & \\
\hline & & $\mathrm{TC}$ & $32(32.98)$ & $54(43.54)$ & 0.24 & 0.61 & $0.85(0.47-1.56)$ \\
\hline & & $\mathrm{CC}^{*}$ & $27(25.77)$ & 15 (12.09) & 6.31 & 0.01 & $2.60(1.22-5.54)$ \\
\hline & & $\mathrm{CC}$ versus $\mathrm{TC}+\mathrm{TT}$ & \# & \# & 0.59 & 0.43 & $1.23(0.72-2.12)$ \\
\hline & & TT versus CC $+\mathrm{TC}$ & \# & \# & $8.71^{*}$ & $0.03^{*}$ & $0.35(0.17-0.71)$ \\
\hline & & $\mathrm{T}$ & $108(55.67)$ & $164(66.1)$ & Reference & & \\
\hline & & $\mathrm{C}$ & $86(44.32)$ & $84(33.8)$ & $5.01^{*}$ & $0.02^{*}$ & $1.55(1.05-2.28)$ \\
\hline
\end{tabular}

${ }^{\#}$ Genotype values are mentioned in above corresponding rows; ${ }^{*}$ values are statistically significant.

TABLE 3: Summary of the interactive combinations of three examined polymorphisms by MDR analysis.

\begin{tabular}{lccc}
\hline Genotype model & $\begin{array}{c}\text { Cross-validation } \\
\text { consistency }\end{array}$ & $\begin{array}{c}\text { Testing } \\
\text { accuracy }\end{array}$ & $P$ value \\
\hline MMEL1, c.1553 T > C & 0.518 & 10 & 0.2061 \\
\hline $\begin{array}{l}\text { MMEL1, c.1553 T > C } \\
+ \text { SH2B3, c.784 T > C }\end{array}$ & 0.641 & 10 & 0.0156 \\
\hline $\begin{array}{l}\text { IRAK1, c.587 A > G } \\
+ \text { SH2B3, c.784 T > C }\end{array}$ & 0.612 & 8 & 0.0786 \\
+ MMEL1, c.1553 T > C & & & \\
\hline
\end{tabular}

The human MMEL1 gene encodes a type II class transmembrane protein (779 amino acids) belonging to metalloendopeptidases family, which is known to show ubiquitous expression. This gene is located on chromosome 1 and its size spans up to $0.4 \mathrm{MB}$ with 24 exons. The genetic polymorphism rs3748816 is located in exon 16 of MMEL1 with the "T" allele codes (ATG) for the nonpolar hydrophobic amino acid methionine and the risk " $\mathrm{C}$ " allele codes (ACG) polar hydrophilic threonine at amino acid position 518 . This change is predicted to be "probably damaging" based on the computational program Polymorphism Phenotyping (PolyPhen) [20]. This amino acid variant is located in alpha helix region close to peptidase family M13 N-terminal domain of MMEL1 protein. The MMEL1 protein in conjunction with other members of the metalloendopeptidase family appears to be involved in neuropeptide degradation [21]. The recent GWAS and follow-up case-control studies have identified the association of MMEL1 gene with rheumatoid arthritis (RA) susceptibility [22-24].

Growing evidence suggests that autoimmune diseases such as celiac disease and rheumatoid arthritis share at least 10 common genetic susceptibility loci [25]. Of those overlapping loci, rs4648562 $\left(r^{2} \geq 0.89\right)$ of MMEL1 gene leads to splice site alteration of the concerned transcript. Although celiac disease and rheumatoid arthritis are distinct diseases by nature, they share common features such as association with HLA status, T-cell infiltration in target organs, and development of autoimmune antibodies. It is not clear what function this gene plays in gluten sensitivity if it is indeed a true disease associated gene. TNFRSF14 (rs2234167), a strong candidate for celiac diseases, which is less than $60 \mathrm{~kb}$ downstream is in strong linkage $\left(r^{2}=0.89\right)$ with rs3890745 of MMEL1 further raising the possibility of its indirect involvement in celiac disease [26]. However, further work involving reverse genetics is required to fully establish the function of this gene and to ascertain how the disease-causative polymorphism(s) in MMEL1 gene exert their effect in celiac disease biology.

Although both IRAK1 (rs1059702) and SH2B3 (rs3184504) nonsynonymous genetic polymorphisms are statistically not associated with CD, this does not mean that they are not true disease susceptibility loci among Saudi Arabian patients. Independently these two loci are seen under strong linkage [by LD scores; $r^{2}>0.9$ ] with genetically critical regions for celiac disease development in Caucasians [14]. On either side (at least $0.2 \mathrm{MB}$ ) of the rs3184504 at 12q24 (Chr 12: 111833788 to Chr 12: 111932800) exist candidate genes like RNA processing factor ATXN2 and the nuclear localization inhibitor 
BRAP, which are known for their role in subclinical inflammation and lymphocyte activity regulation [27], and they were previously associated with CD susceptibility [13]. Indeed, rs3184504 was seen to be associated with expression status of SH2B3 in intestinal mucosa of patients with CD [28]. It is interesting to note that this polymorphism involves an amino acid substitution $\mathrm{R} 262 \mathrm{~W}$ in functionally important domain of protein that is known to be under positive natural selection and critical for efficient response to exogenous antigens [29]. The lack of significant association of rs3184504 in Saudi patients but its association in European groups [13, $16,19]$ suggests the genetic heterogeneity of celiac disease.

We acknowledge some limitations in interpreting the present study results. Firstly, we have only screened 3 nonsynonymous genetic polymorphisms and did not cover the $50+$ remaining SNPs (noncoding SNPs) reported by dense genotyping studies. Second, the missing gene expression data of the three associated genetic markers and their linked genetic regions rather than their own allele or genotype frequencies might indeed be the real etiological players in the pathogenesis of celiac disease. However, the biology of complex diseases is certainly much more complex than a direct SNP altered gene function disease relationship, and we must be very cautious when proposing celiac causal genes and pathogenic mechanisms based only on statistical association. Third, the sample size of this study is not large enough to draw a firm conclusion, such that our findings can be generalized to all Arab population. Thus to validate whether the GWAS findings are useful in identifying the celiac disease relevant etiological genetic markers, we recommend further studies involving larger population, extensive SNP coverage assays, and in vitro functional studies.

\section{Conclusion}

In conclusion, this study reports for the first time that MMEL1 $518 \mathrm{Met} / \mathrm{Thr}$ polymorphism contributes to celiac disease risk among Saudi Arabians, both in single and also in synergistic cooperation with $\mathrm{SH} 2 \mathrm{~B} 3$ gene marker. Taken together, our study results have yielded mixed findings in replicating the European specific CD genetic susceptibility loci among a geographically and culturally distinct population from the Saudi Arabian Peninsula. In light of our results, this study further stresses the importance of identifying populationspecific genetic susceptibility loci to obtain robust results that can be replicated. In particular, replication of GWAS loci association in populations like Saudi Arabians is relevant given their changing food habits with substantial dietary gluten component. To better understand the clinical implications of CD-specific GWAS loci, this study will further establish background data for further investigations into the mechanisms through which MMEL1 is likely to contribute in celiac disease development.

\section{Conflict of Interests}

The authors declare that there is no conflict of interests regarding the publication of this paper.

\section{Authors' Contribution}

Omar I. Saadah and Noor Ahmad Shaik have equally contributed.

\section{Acknowledgments}

All authors sincerely extend their thanks to Princess AlJawhara Al-Brahim Center of Excellence in Research of Hereditary Disorders (PACER-HD) for its support in laboratory logistics required for this study. The authors deeply appreciate Ms. Sana Khadir for her kind effort in coordinating with patients and controls for sampling and clinical data collection. This work was funded by the Deanship of Scientific Research (DSR), King Abdulaziz University, under Grant no. HiCi-1434-140-1. The authors, therefore, acknowledge the DSR technical and financial support.

\section{References}

[1] A. Di Sabatino and G. R. Corazza, "Coeliac disease," The Lancet, vol. 373, no. 9673, pp. 1480-1493, 2009.

[2] A. Fasano and C. Catassi, "Celiac disease," The New England Journal of Medicine, vol. 367, no. 25, pp. 2419-2426, 2012.

[3] A. H. Gudjónsdóttir, S. Nilsson, J. Ek, B. Kristiansson, and H. Ascher, "The risk of celiac disease in 107 families with at least two affected siblings," Journal of Pediatric Gastroenterology and Nutrition, vol. 38, no. 3, pp. 338-342, 2004.

[4] G. Samaşca, G. Sur, and I. Lupan, "Current trends and investigative developments in celiac disease," Immunological Investigations, vol. 42, no. 4, pp. 273-284, 2013.

[5] G. Telega, T. R. Bennet, and S. Werlin, "Emerging new clinical patterns in the presentation of celiac disease," Archives of Pediatrics and Adolescent Medicine, vol. 162, no. 2, pp. 164-168, 2008.

[6] M. A. D’Amico, J. Holmes, S. N. Stavropoulos et al., "Presentation of pediatric celiac disease in the United States: prominent effect of breastfeeding," Clinical Pediatrics, vol. 44, no. 3, pp. 249-258, 2005.

[7] M. Vives-Pi, S. Takasawa, I. Pujol-Autonell et al., "Biomarkers for diagnosis and monitoring of celiac disease," Journal of Clinical Gastroenterology, vol. 47, no. 4, pp. 308-313, 2013.

[8] K. Barada, H. Abu Daya, K. Rostami, and C. Catassi, "Celiac disease in the developing world," Gastrointestinal Endoscopy Clinics of North America, vol. 22, no. 4, pp. 773-796, 2012.

[9] M. B. Hariz, M. Kallel-Sellami, L. Kallel et al., "Prevalence of celiac disease in Tunisia: mass-screening study in schoolchildren," European Journal of Gastroenterology and Hepatology, vol. 19, no. 8, pp. 687-694, 2007.

[10] M. Abu-Zekry, D. Kryszak, M. Diab, C. Catassi, and A. Fasano, "Prevalence of celiac disease in Egyptian children disputes the east-west agriculture-dependent spread of the disease," Journal of Pediatric Gastroenterology and Nutrition, vol. 47, no. 2, pp. 136-140, 2008.

[11] U. Volta and V. Villanacci, "Celiac disease: diagnostic criteria in progress," Cellular and Molecular Immunology, vol. 8, no. 2, pp. 96-102, 2011.

[12] D. A. van Heel, L. Franke, K. A. Hunt et al., "A genome-wide association study for celiac disease identifies risk variants in the region harboring IL2 and IL21," Nature Genetics, vol. 39, no. 7, pp. 827-829, 2007. 
[13] K. A. Hunt, A. Zhernakova, G. Turner et al., "Newly identified genetic risk variants for celiac disease related to the immune response," Nature Genetics, vol. 40, no. 4, pp. 395-402, 2008.

[14] G. Trynka, A. Zhernakova, J. Romanos et al., "Coeliac diseaseassociated risk variants in TNFAIP3 and REL implicate altered NF-kappaB signalling," Gut, vol. 58, no. 8, pp. 1078-1083, 2009.

[15] E. A. M. Festen, P. Goyette, T. Green et al., "A meta-analysis of genome-wide association scans identifies IL18RAP, PTPN2, TAGAP, and PUS10 as shared risk loci for Crohn's disease and celiac disease," PLoS Genetics, vol. 7, no. 1, Article ID e1001283, 2011.

[16] G. Trynka, K. A. Hunt, N. A. Bockett et al., "Dense genotyping identifies and localizes multiple common and rare variant association signals in celiac disease," Nature Genetics, vol. 43, no. 12, pp. 1193-1201, 2011.

[17] P. C. A. Dubois, G. Trynka, L. Franke et al., "Multiple common variants for celiac disease influencing immune gene expression," Nature Genetics, vol. 42, no. 4, pp. 295-302, 2010.

[18] C. P. Garner, J. A. Murray, Y. C. Ding, Z. Tien, D. A. van Heel, and S. L. Neuhausen, "Replication of celiac disease UK genomewide association study results in a US population," Human Molecular Genetics, vol. 18, no. 21, pp. 4219-4225, 2009.

[19] D. A. van Heel, K. Hunt, L. Greco, and C. Wijmenga, "Genetics in coeliac disease," Best Practice and Research: Clinical Gastroenterology, vol. 19, no. 3, pp. 323-339, 2005.

[20] M. Ban, J. L. McCauley, R. Zuvich et al., "A non-synonymous SNP within membrane metalloendopeptidase-like 1 (MMEL1) is associated with multiple sclerosis," Genes and Immunity, vol. 11, no. 8, pp. 660-664, 2010.

[21] J. Y. Huang, A. M. Bruno, C. A. Patel et al., "Human membrane metallo-endopeptidase-like protein degrades both betaamyloid 42 and beta-amyloid 40," Neuroscience, vol. 155, no. 1 , pp. 258-262, 2008.

[22] S. Raychaudhuri, E. F. Remmers, A. T. Lee et al., "Common variants at CD40 and other loci confer risk of rheumatoid arthritis," Nature Genetics, vol. 40, no. 10, pp. 1216-1223, 2008.

[23] A. Barton, W. Thomson, X. Ke et al., "Rheumatoid arthritis susceptibility loci at chromosomes 10p15, 12q13 and 22q13," Nature Genetics, vol. 40, no. 10, pp. 1156-1159, 2008.

[24] P. Danoy, M. Wei, H. Johanna et al., "Association of variants in MMEL1 and CTLA4 with rheumatoid arthritis in the Han Chinese population," Annals of the Rheumatic Diseases, vol. 70, no. 10, pp. 1793-1797, 2011.

[25] M. J. H. Coenen, G. Trynka, S. Heskamp et al., "Common and different genetic background for rheumatoid arthritis and coeliac disease," Human Molecular Genetics, vol. 18, no. 21, pp. 4195-4203, 2009.

[26] H. W. Jung, S. J. La, J. Y. Kim et al., "High levels of soluble herpes virus entry mediator in sera of patients with allergic and autoimmune diseases," Experimental and Molecular Medicine, vol. 35, no. 6, pp. 501-508, 2003.

[27] G. Auburger, S. Gispert, S. Lahut et al., "12q24 locus association with type 1 diabetes: SH2B3 or ATXN2?" World Journal of Diabetes, vol. 5, no. 3, pp. 316-327, 2014.

[28] L. Plaza-Izurieta, A. Castellanos-Rubio, I. Irastorza et al., "Revisiting genome wide association studies (GWAS) in coeliac disease: replication study in Spanish population and expression analysis of candidate genes," Journal of Medical Genetics, vol. 48, no. 7, pp. 493-496, 2011.

[29] A. Zhernakova, C. C. Elbers, B. Ferwerda et al., "Evolutionary and functional analysis of celiac risk loci reveals $\mathrm{SH} 2 \mathrm{~B} 3$ as a protective factor against bacterial infection," American Journal of Human Genetics, vol. 86, no. 6, pp. 970-977, 2010. 


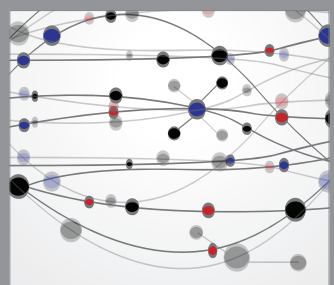

The Scientific World Journal
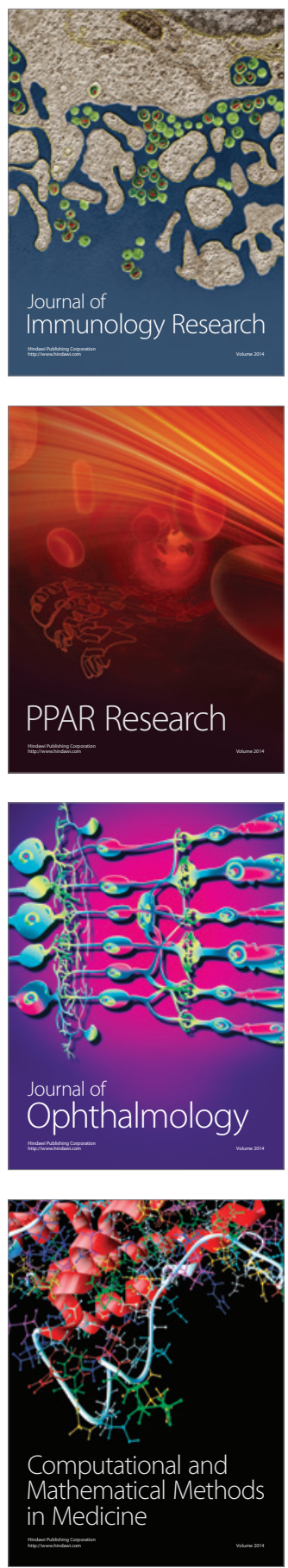

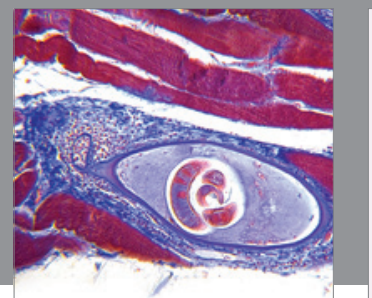

Gastroenterology

Research and Practice
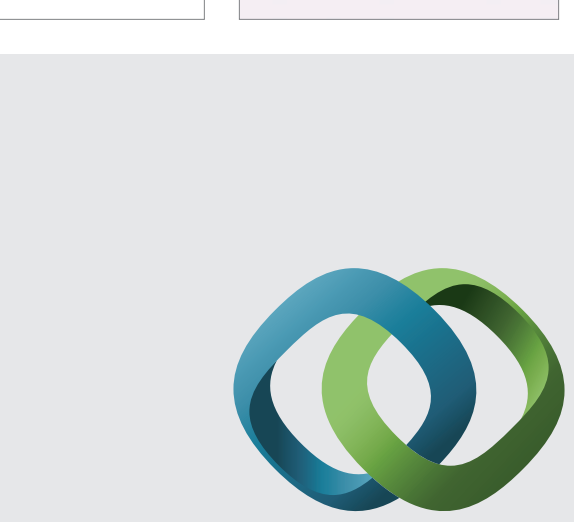

\section{Hindawi}

Submit your manuscripts at

http://www.hindawi.com
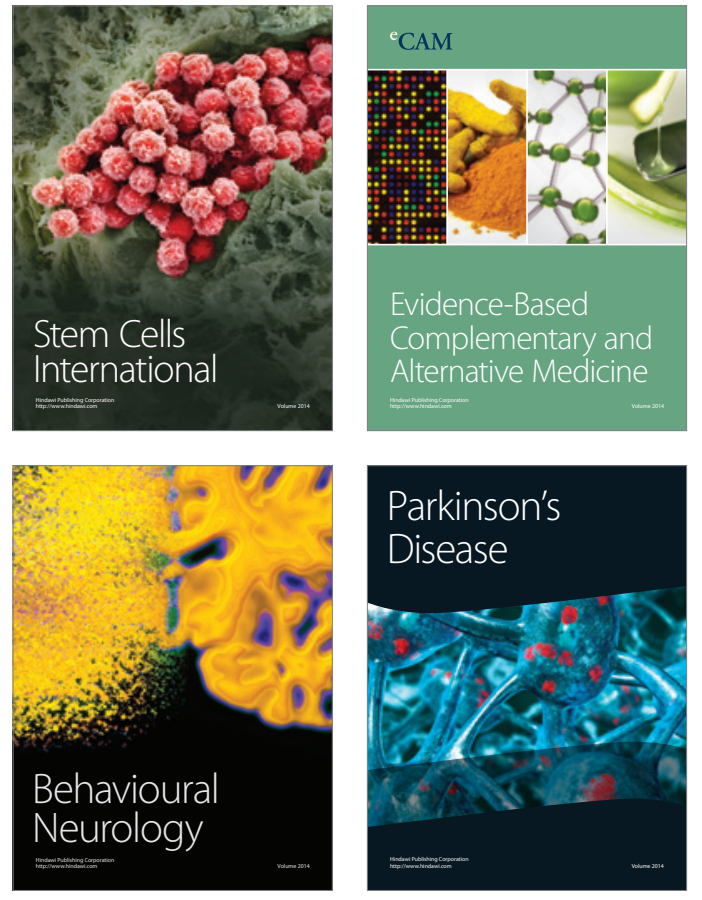
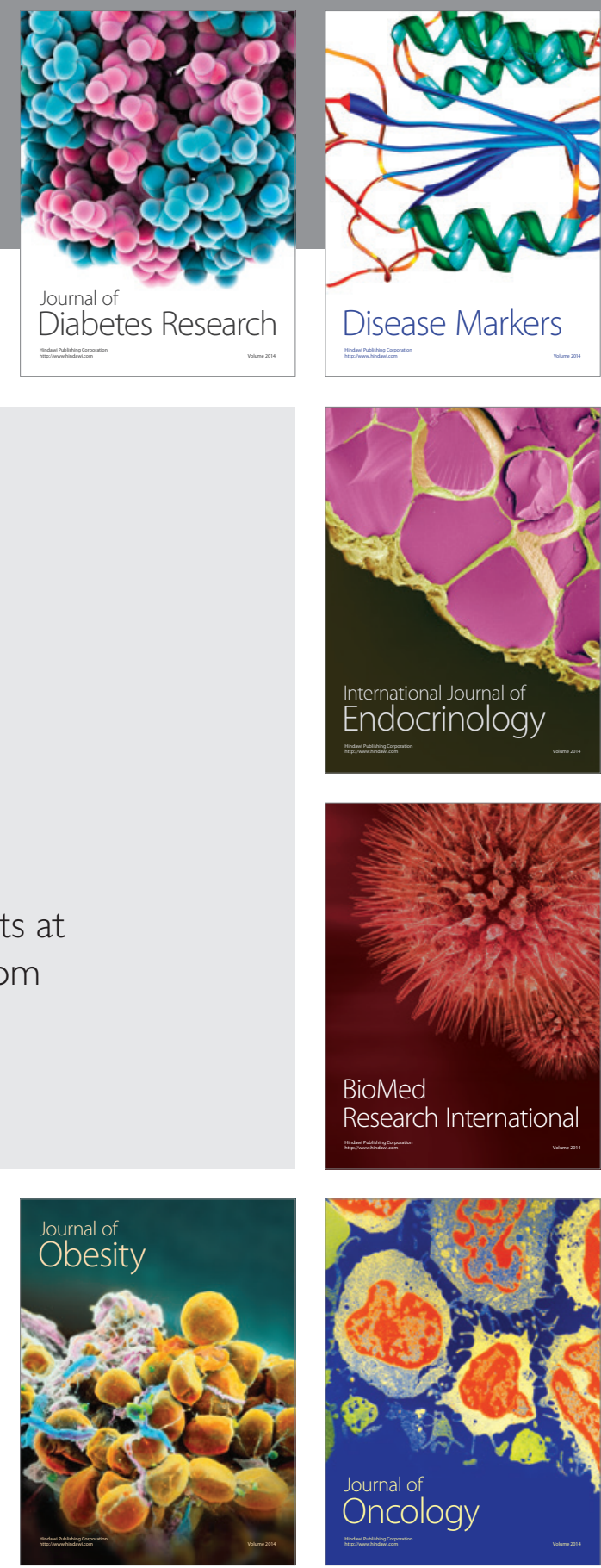

Disease Markers
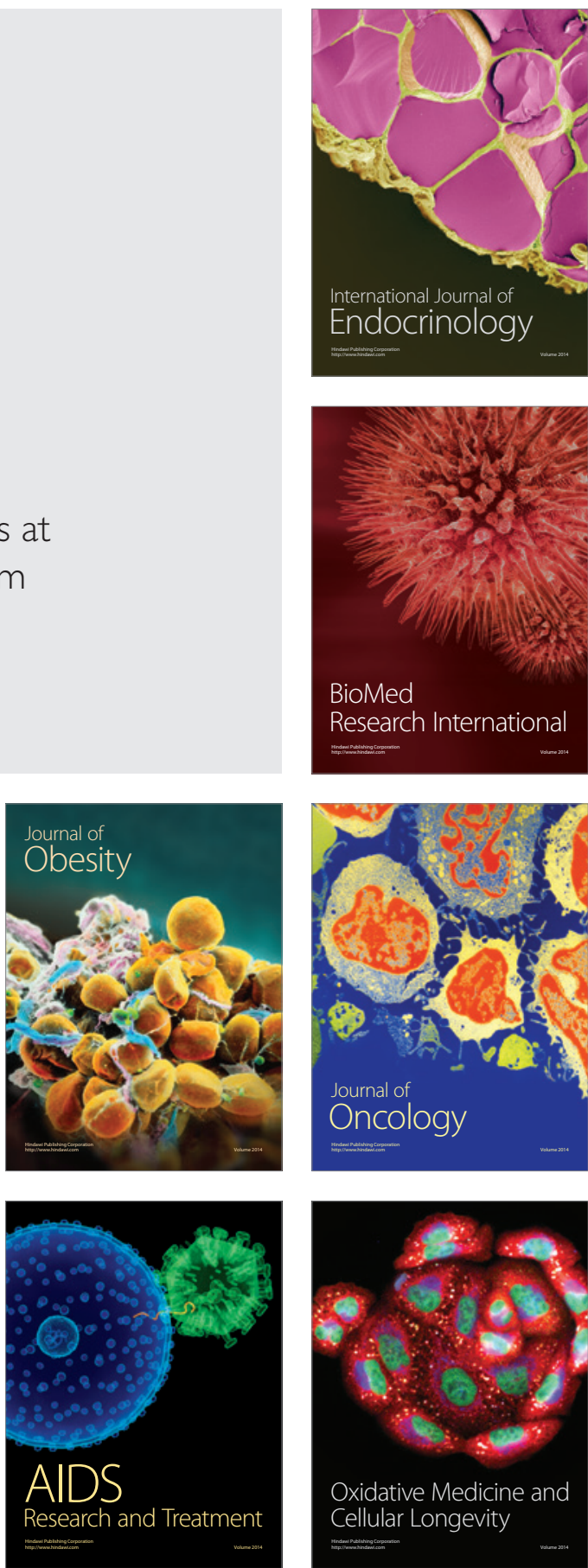